\title{
BMJ Open Protocol for a prospective observational study to develop a frailty index for use in perioperative and critical care
}

\author{
Jai N Darvall, ${ }^{1,2,3}$ Sabine Braat, ${ }^{4,5}$ David A Story, ${ }^{3}$ Kate Greentree, ${ }^{1,2}$ Tony Bose, ${ }^{1,2}$ \\ Joel Loth, ${ }^{1,2}$ Wen K Lim ${ }^{6}$
}

To cite: Darvall JN, Braat S, Story DA, et al. Protocol for a prospective observational study to develop a frailty index for use in perioperative and critical care. BMJ Open 2019;9:e024682. doi:10.1136/ bmjopen-2018-024682

- Prepublication history for this paper is available online. To view these files please visit the journal online (http://dx.doi. org/10.1136/bmjopen-2018024682).

Received 12 June 2018 Revised 29 November 2018 Accepted 29 November 2018

Check for updates

(C) Author(s) (or their employer(s)) 2019. Re-use permitted under CC BY-NC. No commercial re-use. See rights and permissions. Published by BMJ.

For numbered affiliations see end of article.

Correspondence to

Dr Jai N Darvall;

jai.darvall@mh.org.au

\section{ABSTRACT}

Introduction Frailty is of increasing importance to perioperative and critical care medicine, as the proportion of older patients increases globally. Evidence continues to emerge of the considerable impact frailty has on adverse outcomes from both surgery and critical care, which has led to a proliferation of different frailty measurement tools in recent years. Despite this, there remains a lack of easily implemented, comprehensive frailty assessment tools specific to these complex populations. Development of a frailty index using routinely collected hospital data, able to leverage the automated aspects of an electronic medical record, would aid risk stratification and benefit clinicians and patients alike.

Methods and analysis This is a prospective observational study. 150 intensive care unit (ICU) patients aged $\geq 50$ years and 200 surgical patients aged $\geq 65$ years will be enrolled. The primary objective is to develop a frailty index. Secondary objectives include assessing its ability to predict in-hospital mortality and/or discharge to a new non-home location; the performance of the frailty index in predicting postoperative and ICU complications, as well as health-related quality of life at 6 months; to compare the performance of the frailty index against existing frailty measurement and risk stratification tools; and to assess its modification by patients' health assets.

Ethics and dissemination This study has been approved by the Melbourne Health Human Research Ethics Committee(20 January 2017, HREC/16/MH/321). Dissemination will be via international and national anaesthetic and critical care conferences, and publication in the peer-reviewed literature.

\section{INTRODUCTION}

Frailty is increasingly recognised as a clinical entity of importance to clinicians, patients and the health system at large. In particular, the relevance of patient frailty to perioperative and critical care is growing, as older adults comprise an increasing proportion of patients presenting for surgery and to intensive care units (ICUs) worldwide. Over the next decade, adults aged $>65$ years will account for more than one-quarter of all ICU admissions in many countries, and up to half of all surgical patients. ${ }^{1-5}$ In the largest

\section{Strengths and limitations of this study}

This is a prospective, large-scale study in a tertiary metropolitan setting.

- There are limited prospective studies using well-constructed comprehensive frailty indices in the surgical and critical care fields.

- It is not known how frailty indices compare with traditional risk stratification tools in these areas.

- A limitation is that routinely collected hospital data varies slightly between health services, although sensitivity analyses will help to determine the impact of individual variables on results.

ICU study to date, ${ }^{6}$ frailty was associated with almost twice the odds of mortality and new functional dependence, and in the largest meta-analysis of postsurgical complications (encompassing over 12000 surgical patients across 44 studies) frailty was perhaps the most important predictor of adverse outcome, with more than doubled odds of postoperative complications. ${ }^{7}$ In the almost-decade since the 2010 UK National Confidential Enquiry into Patient Outcome and Death (NCEPOD) examined older adults undergoing surgery, the recommendation that "An agreed means of assessing frailty in the perioperative period should be developed and included in risk assessment' remains unfulfilled. ${ }^{8}$

Frailty can be described as either a phenotypic construct (criteria such as exhaustion, weakness, low activity or weight loss are present) or as a deficit model (risk accumulates due to impairments in health-related domains such as medical comorbidities, cognition, mood and behaviour, communication, sensorium, continence, nutrition and medications). ${ }^{910}$ Although various frailty measurement tools have been devised, these can be of limited use in the ICU or surgical setting. Performance-based measures, for example, such as 'timed-up-and-go' tests or grip strength assessment are impossible for 
mechanically ventilated or acutely unwell patients to perform. ${ }^{11}$ Similarly, assessments including subjective questions requiring patient input may not be possible in this cohort, with potential for error when information is derived from surrogates. ${ }^{12}$ Conversely, the perioperative and ICU environments are incredibly data-rich, with much information collected routinely for all admitted patients. Particularly in the era of electronic medical records, this has the potential to allow automated data integration and rapid derivation of relevant risk stratification scores.

In addition to potential automated calculation, a frailty index based on accumulated deficits has other advantages. As described by Searle $e t$ al, frailty indices demonstrate reproducibility across different populations, despite differences in composition related to which individual health deficits are present in a particular index. ${ }^{13}$ As such, as long as certain prerequisites regarding candidate deficits are met (at least 30 deficits should be included, they should be associated with health status, increase in prevalence with age, not saturate too early, cover a range of systems and remain constant if intended for repeated use in the same population), subtle variation in terminology or specific data collected between health services for use in a frailty index do not matter.

The objective of this study, then, is to develop a frailty index, based on accumulated health deficits, that is able to be incorporated into routine hospital management of ICU and surgical patients.

\section{AIMS}

The primary aim of this study is to develop a frailty index based on accumulated health deficits, using routinely collected hospital data, enabling rapid and easily scalable assessment of surgical and ICU patients' frailty. Secondary aims are to assess the performance of the frailty index in predicting in-hospital mortality and/or discharge to a new non-home location, postoperative and ICU complications, as well as health-related quality of life at 6 months; to compare the performance of the frailty index against existing frailty measurement and risk stratification tools; and to assess its modification by patients' health assets (protective factors that support health and well-being).

\section{METHODS}

\section{Study design}

This study is designed as a prospective, single-centre cohort study, with follow-up period 6 months postdischarge.

\section{Study setting}

This study will be conducted at the Royal Melbourne Hospital (RMH), Melbourne, Australia, a tertiary metropolitan hospital that admits over 2000 ICU patients and with a surgical volume of over 25000 operations annually. Enrolment and follow-up are expected between February 2017 and December 2018.

\section{Inclusion and exclusion criteria}

Patients are eligible if they are:

- Aged $\geq 65$ years on admission for any surgery (emergency or elective).

- Aged $\geq 50$ years on admission to the ICU for any indication.

- Provide written informed consent (or the person responsible in the event of incapacitation).

Patients are ineligible if they are:

- Non-English speaking (or the person responsible is non-English speaking).

- Admitted to the ICU or operating theatre for reasons of organ retrieval.

\section{Data collection (routine for all patients)}

Baseline demographics: preoperatively (surgical patients) or on admission to the ICU (ICU patients): age, gender, height and weight will be collected.

Surgical data: operative type (surgical specialty)/severity (defined according to the P-POSSUM scoring system), blood loss and American Society of Anesthesiology score will be recorded.

ICU data: routine ICU data collected (relating to the entire ICU admission episode) will include mechanical ventilation, renal replacement therapy, cardiac arrest, inotropes/vasoactive infusions, Acute Physiology and Chronic Health Evaluation (APACHE) III illness severity score and presence of any limitations to medical treatment.

Other hospital data: routine data recorded on admission for RMH patients (and common to most health services) will be used to generate a frailty index, consisting of 36 health deficits (table 1). Data will be derived from the falls risk assessment and management plan (RMH form designation: form IP8L), malnutrition risk assessment and management plan (IP63C), pressure injury prevention plan (IP8G), daily nursing care plan (IP8F), and nursing admission and assessment (IP8). Chosen deficits increase in prevalence with age and encompass a range of systems associated with health status.

\section{Data collection (additional, by study investigators)}

Additional admission data: the Katz Index of independence in activities of daily living and Charlson comorbidity score will be collected, with information added to the frailty index (table 1) below. Data related to surgical risk stratification will be collected, including the P-POSSUM Score, ${ }^{14}$ albumin and lactate (where available), and will be compared with the frailty index for prediction of secondary outcomes listed.

Other frailty measurements: the Clinical Frailty Score ${ }^{10}$ and the Edmonton Frailty Score ${ }^{15}$ will both be collected. For patients who are unable to perform the 'Timed Up and Go Test' component of the latter scale (eg, emergency surgical or mechanically ventilated ICU patients), a Reported Edmonton score will be derived. ${ }^{16}$ Data collected represent the health status of the patient prior to the onset of acute illness. 
Table 1 Frailty Index (from routine data collection)

\begin{tabular}{|c|c|c|}
\hline 1 & Falls in last 12 months & $0=$ no, $1=$ yes \\
\hline 2 & Dementia diagnosis & $0=$ no, $1=$ yes \\
\hline 3 & Altered cognition & $0=$ no, $1=$ yes \\
\hline 4 & $\begin{array}{l}\text { On four or more medications, at least } \\
\text { one affecting CNS/CVS }\end{array}$ & $0=$ no, $1=$ yes \\
\hline 5 & Vision impairment & $0=$ no, $1=$ yes \\
\hline 6 & Hearing impairment & $0=$ no, $1=$ yes \\
\hline 7 & Assistance with transferring & $0=$ no, $1=y e s$ \\
\hline 8 & Assistance with mobilising & $0=$ no, $1=$ yes \\
\hline 9 & Assistance with toileting & $0=$ no, $1=$ yes \\
\hline 10 & Assistance with bathing & $0=$ no, $1=$ yes \\
\hline 11 & Assistance with dressing & $0=$ no, $1=y e s$ \\
\hline 12 & Postural hypotension/dizziness & $0=$ no, $1=$ yes \\
\hline 13 & Bowel incontinence & $0=$ no, $1=$ yes \\
\hline 14 & Urinary incontinence & $0=$ no, $1=$ yes \\
\hline 15 & Eating poorly? & $0=$ no, $1=$ yes \\
\hline 16 & Lost weight without trying? & $\begin{array}{l}0=\text { no, } 0.5=1-10 \mathrm{~kg}, \\
1=>10 \mathrm{~kg}\end{array}$ \\
\hline 17 & Pressure injury- current or past & $0=$ no, $1=$ yes \\
\hline 18 & Neuropathic foot disease & $0=$ no, $1=$ yes \\
\hline 19 & $\begin{array}{l}\text { Problems managing at home prior to } \\
\text { admission }\end{array}$ & $0=$ no, $1=$ yes \\
\hline
\end{tabular}

$20 \begin{aligned} & \text { Often feels sad or depressed? } \\ & \text { (Edmonton) }\end{aligned}$

21 Requires assistance with eating? $\quad 0=$ no, $1=y e s$ (Katz)†

\begin{tabular}{|c|c|c|}
\hline & Charlson Comorbidity Datał & \\
\hline 22 & Ischaemic heart disease & $0=$ no, $1=y e s$ \\
\hline 23 & Congestive heart failure & $0=$ no, $1=y e s$ \\
\hline 24 & Peripheral vascular disease & $0=$ no, $1=y e s$ \\
\hline 25 & Cerebrovascular disease & $0=$ no, $1=$ yes \\
\hline 26 & Hemiplegia & $0=$ no, $1=y e s$ \\
\hline 27 & Chronic lung disease & $0=$ no, $1=y e s$ \\
\hline 28 & Connective tissue disease & $0=$ no, $1=y e s$ \\
\hline 29 & Peptic ulcer disease & $0=$ no, $1=y e s$ \\
\hline 30 & Chronic liver disease & $0=$ no, $1=y e s$ \\
\hline 31 & Diabetes & $0=$ no, $1=y e s$ \\
\hline 32 & Leukaemia/lymphoma & $0=$ no, $1=y e s$ \\
\hline 33 & Malignant tumour & $0=$ no, $1=y e s$ \\
\hline 34 & Metastatic cancer & $0=$ no, $1=y e s$ \\
\hline 35 & Moderate/severe kidney disease & $0=$ no, $1=y e s$ \\
\hline \multirow[t]{2}{*}{36} & Moderate/severe liver disease & $0=$ no, $1=$ yes \\
\hline & & Total $=$ \\
\hline
\end{tabular}

*Derived from the Edmonton Frail Scale.

†Derived from the Katz Index of independence in activities of daily living.

$\ddagger$ Derived from the Charlson Comorbidity Index.

CNS, central nervous system; CVS, cardiovascular system.

Health asset data: the health assets index developed by Gregorevic et $a l^{17}$ will be used, with data collected including educational level, family proximity, financial means, social engagement and psychosocial well-being (representing patients' baseline state prior to hospital admission).

Outcomes: endpoints collected will include in-hospital mortality (primary outcome); length of stay (time in days between either admission to the intensive care unit and discharge from hospital, or surgical operation and discharge from hospital); discharge destination (including new non-home discharge, including assisted living facility, rehabilitation or other acute hospital location); and postoperative/ICU complications (acute myocardial infarction, cardiac arrest, sepsis, acute pulmonary oedema, deep venous thrombosis, pulmonary embolism, stroke/transient ischaemic attack, wound infection, unplanned return to operating theatre, unplanned ICU/ HDU admission) (all secondary outcomes). The outcome assessors will have access to frailty information collected.

6-month follow-up: health-related quality of life (EQ-5D scale), place of residence (home, residential care facility and hospital) and Clinical Frailty Scale will be recorded. A scripted telephone text will be used, proven feasible and valid in both geriatric populations and ICU survivors. ${ }^{18} 19$ In addition, we believe that this study will be the first to administer the Clinical Frailty Scale by telephone, thus will provide an assessment of its feasibility through this modality.

\section{Statistical analyses}

We will calculate a frailty index score for each patient with $\geq 80 \%$ non-missing health deficit scores. ${ }^{20} \mathrm{~A}$ frailty index score will be derived for each patient as the sum of the deficit scores divided by the total number of non-missing deficit scores thus ranging from 0 (no deficits) to 1 (all deficits). Patients with a frailty index score of $\geq 0.25$ will be considered frail. ${ }^{21}$ A histogram and descriptive statistics of the frailty index scores will be provided for the entire patient sample and by surgical/ICU patients, gender and age. Mean frailty index scores will be plotted versus age for all patients and by surgical/ICU status. A linear regression analysis of the frailty index on age will be performed and by surgical/ICU status to obtain the rate of accumulation of health deficits over age, in case of positive skewness, the frailty index scores may be log transformed before analysis. A random sampling procedure using $80 \%$ of frailty index items without replacement will be used and repeated several times to investigate the impact of an individual item on the rate. This approach has been successfully used in similar studies of frailty indices. ${ }^{22}$

A logistic regression model will be fitted to the outcomes of in-hospital mortality (primary outcome), discharge to a new non-home location, in-hospital mortality or discharge to a new non-home location, postoperative and ICU complications, including in the model the frailty index, surgical/ICU status, gender and age. Receiver operating characteristic (ROC) curves and the area under the ROC curves will be obtained to assess the ability of the model to discriminate between two classes of these outcomes. Health-related quality of life at 6 months, whereby death will be coded as 0 , will be analysed with 
a linear regression model using the explained variation to evaluate overall model performance. Anticipating about $5 \%$ missing quality of life data, we will use multiple imputations to explore the sensitivity of the results to underlying missing data assumptions. We will obtain Spearman's rank correlation between the frailty measurement tools (the frailty index, Edmonton and Clinical Frailty Scales) on admission and between the frailty index and risk stratification tools (APACHE for ICU and P-POSSUM for surgery). Each patient's score will be categorised into frail, vulnerable and non-frail (reference); for the frailty index (frail $\geq 0.25$, vulnerable $0.2-<0.25$, non-frail $0-<0.2$ ), Edmonton (frail $\geq 8$, vulnerable 6-7, non-frail 0-5) and Clinical Frailty Scale (frail $\geq 5$, vulnerable 4, non-frail 1-3). We will examine the difference in the strength in an association between each categorised frailty scale and the outcomes using the models described earlier. Furthermore, we will obtain the measures listed above to compare the ability of the models to predict these outcomes between the three categorised frailty tools and to predict in-hospital mortality between the frailty index and risk stratification tools. Modification of the effect in frail versus non-frail patients by health assets for outcomes (death, new non-home discharge location, and postoperative and ICU complications) will be examined for the frailty index using interaction tests.

\section{Sample size}

A convenience sample of 200 surgical and 150 ICU patients from a single hospital is planned. Based on comparative literature (including a systematic review and meta-analysis of over 8000 surgical patients, and the largest multicentre study of ICU frailty), we assume that $20 \%$ of surgical patients and $30 \%$ of ICU patients, combined about $24 \%$, will be frail. ${ }^{623}$ We will be able to obtain a $95 \%$ CI of $\pm 4.4 \%$ around the prevalence of frailty of $24 \%$ with a sample size of 350 . In addition, we anticipate an in-hospital mortality of $5 \%$ in surgical patients (based on the pooled mortality rate in the meta-analysis above) and $21 \%$ in ICU patients, overall about $10 \%$. Assuming the odds of in-hospital mortality of frail patients is 3.5 times than that of non-frail patients (based on pooled ORs from previous systematic reviews ${ }^{23} 24$ ) and in-hospital mortality is $6.8 \%$ in those who are not frail, the power to detect this effect with a sample size of 350 patients is $87 \%$ (two-sided 5\% alpha).

\section{Patient and public involvement}

The development of the study design was informed by patient-centred endpoints-rather than just assessing mortality, new residential care admission, postoperative and post-ICU complications are outcome measures of importance to patients. Similarly, assessment of the quality of life (and potential decrement in quality of life) is also patient-centred. During the consent process, patients or their person responsible are given verbal and written information that study results are available to be disseminated to them. A written summary of the study findings will be provided in this instance. Specific patients were not however formally involved in the study design.

\section{DISCUSSION}

The importance of routine frailty assessment in the perioperative and ICU setting has been emphasised by various organisations, including the American College of Surgeons, the American Geriatrics Society and the Association of Anaesthetists of Great Britain and Ireland. ${ }^{25} 26$ Given this pressing need for an easily implementable and valid frailty assessment tool, various scales have been developed. One such frailty measure, the 'modified frailty index' ( $\mathrm{mFI})$, has the advantage of using automatically collected variables from the US National Surgical Quality Improvement Program (NSQIP) database. Unfortunately, only 11 items are contained in this index, with the majority (9) representing medical comorbidities, thus it can perhaps be more accurately described as predominantly a 'comorbidity' scale. ${ }^{27}$ The Groningen frailty scale, although encompassing a wider range of health deficits, numbers only 15 deficits in total. ${ }^{28}$ A third proposed scale, the Johns Hopkins Adjusted Clinical Groups frailty-defining diagnoses indicator, only comprises 12 items and includes criteria such as 'poverty' and 'barriers to access to care' which may more accurately be described as absent health assets, rather than deficits contributing to frailty per se. ${ }^{29}$ More recently, frailty indices in non-surgical/ICU specific populations have been developed using other health databases such as Medicare claimsbased data, ${ }^{30}$ or the inter-RAI assessment system, which aids the comprehensive geriatric assessment of older hospitalised inpatients. ${ }^{22}$

This study will thus provide a comprehensive and timely assessment measure of frailty, and its importance in an increasingly elderly surgical and critically ill population. Although routinely collected data do vary slightly between health services, potentially limiting generalisability, conducting sensitivity analyses will allow for an individual variable effect to be assessed. As this group poses new challenges for anaesthetists, intensivists, surgeons, perioperative physicians and health services alike, derivation of an automated frailty index using routine hospital data has the potential to revolutionise risk stratification and improve outcomes, as the prevalence of frailty increases dramatically in coming years.

Author affiliations

${ }^{1}$ Department of Intensive Care, Royal Melbourne Hospital, Melbourne, Victoria, Australia

${ }^{2}$ Department of Anaesthesia and Pain Management, Royal Melbourne Hospital, Melbourne, Victoria, Australia

${ }^{3}$ Centre for Integrated Critical Care, University of Melbourne, Melbourne, Victoria, Australia

${ }^{4}$ Centre for Epidemiology and Biostatistics, Melbourne School of Population and Global Health, University of Melbourne, Melbourne, Victoria, Australia

${ }^{5}$ Melbourne Clinical and Translational Science Platform, Melbourne School of

Population and Global Health, University of Melbourne, Melbourne, Victoria, Australia

${ }^{6}$ Department of Medicine, University of Melbourne, Melbourne, Victoria, Australia 
Contributors JND, DAS, SB and WKL: involved in study design, protocol drafting and manuscript preparation. KG, JL and TB: involved in protocol drafting and manuscript proofing.

Funding This work has been partially supported by an Emerging Researcher Scholarship for the first author by the Australian and New Zealand College of Anaesthetists, from a grant from Dr Peter Lowe.

Competing interests None declared.

Patient consent for publication Not required.

Ethics approval Melbourne Health Human Research Ethics Committee .

Provenance and peer review Not commissioned; externally peer reviewed.

Open access This is an open access article distributed in accordance with the Creative Commons Attribution Non Commercial (CC BY-NC 4.0) license, which permits others to distribute, remix, adapt, build upon this work non-commercially, and license their derivative works on different terms, provided the original work is properly cited, appropriate credit is given, any changes made indicated, and the use is non-commercial. See: http://creativecommons.org/licenses/by-nc/4.0/.

\section{REFERENCES}

1. Corke C, Leeuw E, Lo SK, et al. Predicting future intensive care demand in Australia. Crit Care Resusc 2009;11:257-60.

2. Needham DM, Bronskill SE, Calinawan JR, et al. Projected incidence of mechanical ventilation in Ontario to 2026: preparing for the aging baby boomers. Crit Care Med 2005;33:574-9.

3. Reinikainen M, Uusaro A, Niskanen M, et al. Intensive care of the elderly in Finland. Acta Anaesthesiol Scand 2007;51:522-9.

4. Australian Institute of Health and Welfare. Admitted patient care 2015-16: Australian hospital statistics. Health services series no.75. Cat. no. HSE 185 ed. Canberra: Australian Institute of Health and Welfare, 2017.

5. Makary MA, Segev DL, Pronovost PJ, et al. Frailty as a predictor of surgical outcomes in older patients. J Am Coll Surg 2010;210:901-8.

6. Bagshaw S, Ibrahim Q, Majumdar S, et al. Association between frailty and short and long-term outcomes in critically ill patients. Crit Care Med 2013;1:A132.

7. Watt J, Tricco AC, Talbot-Hamon C, et al. Identifying older adults at risk of harm following elective surgery: a systematic review and meta-analysis. BMC Med 2018;16:2.

8. National Confidential Enquiry into Patient Outcome and Death. Elective \& emergency surgery in the elderly: an age old problem. London: NCEPOD, 2010.

9. Fried LP, Tangen CM, Walston J, et al. Frailty in older adults: evidence for a phenotype. J Gerontol A Biol Sci Med Sci 2001;56:M146-M157.

10. Rockwood K, Song X, MacKnight C, et al. A global clinical measure of fitness and frailty in elderly people. CMAJ 2005;173:489-95.

11. Hubbard RE, O'Mahony MS, Woodhouse KW. Characterising frailty in the clinical setting-a comparison of different approaches. Age Ageing 2009;38:115-9.

12. Fisher $\mathrm{C}$, Karalapillai DK, Bailey M, et al. Predicting intensive care and hospital outcome with the Dalhousie Clinical Frailty Scale: a pilot assessment. Anaesth Intensive Care 2015;43:361-8.
13. Searle SD, Mitnitski A, Gahbauer EA, et al. A standard procedure for creating a frailty index. BMC Geriatr 2008;8:24.

14. Prytherch DR, Whiteley MS, Higgins B, et al. POSSUM and Portsmouth POSSUM for predicting mortality. Physiological and operative severity score for the enumeration of mortality and morbidity. Br J Surg 1998;85:1217-20.

15. Rolfson DB, Majumdar SR, Tsuyuki RT, et al. Validity and reliability of the Edmonton Frail Scale. Age Ageing 2006;35:526-9.

16. Hilmer SN, Perera V, Mitchell S, et al. The assessment of frailty in older people in acute care. Australas J Ageing 2009;28:182-8.

17. Gregorevic KJ, Lim WK, Peel NM, et al. Are health assets associated with improved outcomes for hospitalised older adults? A systematic review. Arch Gerontol Geriatr 2016;67:14-20.

18. de Rooij SE, Govers AC, Korevaar JC, et al. Cognitive, functional, and quality-of-life outcomes of patients aged 80 and older who survived at least 1 year after planned or unplanned surgery or medical intensive care treatment. J Am Geriatr Soc 2008;56:816-22.

19. Vest MT, Murphy TE, Araujo KL, et al. Disability in activities of daily living, depression, and quality of life among older medical ICU survivors: a prospective cohort study. Health Qual Life Outcomes 2011;9:9.

20. Hoogendijk EO, Theou O, Rockwood K, et al. Development and validation of a frailty index in the Longitudinal Aging Study Amsterdam. Aging Clin Exp Res 2017;29:927-33.

21. Rockwood K, Mitnitski A. Frailty in relation to the accumulation of deficits. J Gerontol A Biol Sci Med Sci 2007;62:722-7.

22. Hubbard RE, Peel NM, Samanta M, et al. Derivation of a frailty index from the interRAl acute care instrument. BMC Geriatr 2015;15:27.

23. Oakland K, Nadler R, Cresswell L, et al. Systematic review and metaanalysis of the association between frailty and outcome in surgical patients. Ann R Coll Surg Engl 2016;98:80-5.

24. Lin HS, Watts JN, Peel NM, et al. Frailty and post-operative outcomes in older surgical patients: a systematic review. BMC Geriatr 2016;16:157.

25. Chow WB, Rosenthal RA, Merkow RP, et al. Optimal preoperative assessment of the geriatric surgical patient: a best practices guideline from the American College of Surgeons National Surgical Quality Improvement Program and the American Geriatrics Society. $J$ Am Coll Surg 2012;215:453-66.

26. Griffiths R, Beech F, Brown A, et al. Peri-operative care of the elderly 2014: Association of Anaesthetists of Great Britain and Ireland. Anaesthesia 2014;69(Suppl 1):81-98.

27. Velanovich V, Antoine $\mathrm{H}$, Swartz $\mathrm{A}$, et al. Accumulating deficits model of frailty and postoperative mortality and morbidity: its application to a national database. J Surg Res 2013;183:104-10.

28. Steverink N, Slaets J HS, et al. Measuring frailty: developing and testing of the Groningen Frailty Indicator (GFI). Gerontologist 2001;41:236-7.

29. Mclsaac DI, Bryson GL, van Walraven C. Association of frailty and 1-year postoperative mortality following major elective noncardiac surgery: a population-based cohort study. JAMA Surg 2016;151:538-45.

30. Segal JB, Huang J, Roth DL, et al. External validation of the claimsbased frailty index in the national health and aging trends study cohort. Am J Epidemiol 2017;186:745-7. 